ARQGA / 925

\title{
MICROCRISTAIS BILIARES NA PANCREATITE AGUDA IDIOPÁTICA: indício para etiologia biliar oculta subjacente ${ }^{+}$
}

\author{
Júlio Maria Fonseca CHEBLI* Ângelo Paulo FERRARI Jr.* ${ }^{* *}$, Maria Regina Regis SILVA ${ }^{* * *}$, \\ Durval Rosa BORGES $^{* * * *}$, Álvaro Nagib ATALLAH ${ }^{* * * *}$ e Manoel Martins das NEVES ${ }^{* * * * * *}$
}

RESUMO - As principais causas de inflamação pancreática no mundo são a litíase biliar e o alcoolismo crônico. Admite-se que 10 a 30\% das pancreatites agudas sejam idiopáticas. Sugere-se que parte destas são causadas por microlitíase ou barro biliar, identificados pela presença de microcristais no sedimento biliar. Neste estudo, realizou-se análise microscópica da bile obtida por colangiopancreatografia endoscópica, em pacientes com pancreatite aguda idiopática, pancreatite aguda biliar e pancreatite crônica alcoólica - 20 em cada grupo. Pacientes com pancreatite aguda idiopática e microcristais na bile foram submetidos a colecistectomia. Naqueles inaptos à cirurgia efetuou-se esfincterotomia endoscópica ou tratamento com ácido ursodesoxicólico. Pacientes com pancreatite aguda idiopática sem cristais não receberam tratamento específico. A prevalência de microcristais biliares em pacientes com pancreatite aguda idiopática (75\%) e pancreatite aguda biliar (90\%) foi significativamente maior que naqueles com pancreatite crônica alcoólica (15\%). A deteç̧ão de microcristais apresentou sensibilidade de $90 \%$, especificidade de $85 \%$, valor preditivo positivo de $85,7 \%$, valor preditivo negativo de $89,4 \%$ e acurácia de 87,5\% em identificar pancreatite de origem biliar. Nos pacientes com pancreatite aguda idiopática recurrente, cursando com microcristais, houve redução significante dos episódios de pancreatite após tratamento especifico. No seguimento deste grupo durante 23,3 meses, recidiva ocorreu apenas naqueles que apresentavam "fator biliar persistente" (coledocolitíase ou microcristais). Todos os pacientes com pancreatite aguda idiopática submetidos a colecistectomia apresentavam colecistite crônica, e microlitíase foi observada em um paciente. No seguimento ultra-sonográfico, colelitíase foi detectada em um dos casos. No subgrupo de cinco pacientes com pancreatite aguda idiopática sem microcristais houve uma recidiva. Estudo ultrasonográfico durante o seguimento não revelou cálculo biliar em nenhum destes. Concluiu-se que a detecção de microcristais biliares na pancreatite aguda idiopática sugeriu etiologia biliar oculta subjacente. Adicionalmente, intervenção terapêutica especifica nos pacientes com pancreatite aguda idiopática recurrente e microcristais reduziu as recidivas durante o seguimento. Finalmente, pancreatite aguda (particularmente, recurrente) não deveria ser rotulada como idiopática antes da análise microscópica da bile visando a detecção ou exclusão da presença de microcristais.

DESCRITORES - Pancreatite. Colelitíase. Colecistite. Bile. Barro biliar.

Trabalho realizado na Disciplina de Gastroenterologia da Universidade Federal de São Paulo - UNIFESP, São Paulo, SP.

Professor Adjunto da Disciplina de Gastroenterologia da Universidade Federal de Juiz de Fora, MG.

Doutor em Gastroenterologia pela Universidade Federal de São Paulo, Escola Paulista de Medicina - UNIFESP.

** Professor Adjunto da Disciplina de Gastroenterologia da UNIFESP.

*** Professor Adjunto da Disciplina de Patologia Clínica da UNIFESP.

**** Professor Titular da Disciplina de Gastroenterologia da UNIFESP.

***** Professor Titular da Disciplina de Clínica Médica da UNIFESP.

$* * * * * *$ Professor Livre Docente da Disciplina de Gastroenterologia da UNIFESP (In memoriam).

Endereço para correspondência: Dr. Júlio M. Fonseca Chebli - Rua Monsenhor Gustavo Freire, 779/201 - 36016-470 - Juiz de Fora, MG. 


\section{INTRODUÇÃO}

As principais causas de inflamação pancreática no mundo são a litíase biliar e o alcoolismo, sendo estes dois fatores responsáveis por 60 a $80 \%$ dos casos de pancreatite ${ }^{(24,42)}$. Calcula-se que 10 a $30 \%$ das pancreatites aguda sejam idiopáticas ${ }^{(34,42)}$; obviamente esta proporção será afetada pela profundidade da investigação diagnóstica empreendida. É de grande relevância a tendência à recurrência destas formas de pancreatite aguda (PA), do que resulta elevada taxa de morbi-mortalidade ${ }^{(18,37)}$.

É fundamental que se estabeleça a etiologia da PA por sua implicação prognóstica e por facultar opções terapêuticas que melhorem a evolução da doença e evitem ou reduzam o risco de recidivas. Acerca deste ponto, parece avultar em importância o estudo microscópio da bile, objetivando a detecção de microcristais de monohidrato de colesterol (MMC) e bilirrubinato de cálcio (MBC).

Os MMC e MBC foram os primeiros elementos cristalinos identificados na microscopia biliar e reconhecidos como tendo significado patológico no sedimento biliar, geralmente sugestivos de colelitíase, mesmo quando cálculos não são detectados por procedimentos diagnósticos convencionais ${ }^{(4,32)}$. Por sua vez, cristais biliares estão ausentes ou são raros na bile de indivíduos normais ${ }^{(14}$, $23,35,38)$.

Diversos métodos de coleta de bile podem ser utilizados para análise de microcristais. A aspiração de secreções biliares do duodeno, realizada por endoscopia ou por intubação duodenal, precedida por infusão venosa de colecistocinina, é a técnica mais tradicional ${ }^{(4,22,}$ 27). Uma importante limitação desta reside na substancial taxa de falso-negativo para identificação de cálculos ou microcálculos, variando de $30 \%$ a $43 \%{ }^{(14,23,37,44)}$. Tal fato decorre da diluição da bile por secreções digestivas, da presença intermitente de cristais na bile duodenal em muitos casos de colelitíase e da contração incompleta da vesícula em resposta a colecistocinina ${ }^{(23)}$.

A obtenção de bile durante a colangiopancreatografia retrógrada endoscópica (CPRE) pode ser vantajosa. Por coletar bile diretamente do colédoco, a diluição com secreções digestivas não ocorreria. Este fato advoga a possibilidade de haver menor taxa de falso-negativo na identificação de microcristais quando a bile é coletada por este método. Aliado ao fato da CPRE ser procedimento obrigatório na investigação etiológica das pancreatites, especialmente nas formas idiopática e/ou recurrente ${ }^{(30,34)}$, época na qual a coleta de bile já poderia ser realizada, tais ponderações sugerem ser este método o que mais se aproxima do ideal à obtenção de bile para análise de microcristais, pelo menos no tocante aos pacientes com pancreatite.

Inúmeros estudos demonstraram que o atraso no diagnóstico e, por conseguinte, no tratamento da etiologia biliar de uma pancreatite, pode resultar em recurrências muitas vezes fatais em 33 a $48 \%$ dos pacientes, antes de uma segunda admissão hospitalar ${ }^{(15,29,33)}$. Percebe-se, portanto, a importância da detecção de litíase biliar durante um ataque de PA o mais precocemente possível, preferencialmente durante a mesma admissão hospitalar. Neste caso, colecistograma oral mostrou-se não-confiável durante um ataque de pancreatite devido a não opacificação transitória da vesícula biliar ${ }^{(2)}$. Da mesma forma, o ultra-som e mesmo a CPRE podem falhar em demonstrar cálculos biliares ${ }^{(11,26)}$. Isto é explicável pela tendência de muitos pacientes com pancreatite aguda biliar (PAB) apresentarem microcálculos não detectáveis por métodos convencionais de estudo da via biliar ${ }^{(3,7,13)}$. Adicionalmente, verificou-se que proporção significante de doentes com PA, classificada inicialmente como idiopática (PAI), na verdade tem litíase ou microlitíase biliar associada, não detectadas por métodos diagnósticos usuais ${ }^{(9,21,25)}$. Nestas circunstâncias, a detecção de cálculos ou microcálculos na PA pode ser melhorada pela microscopia da bile ${ }^{(27)}$, baseado no fato conhecido de indivíduos formadores de cálculos apresentarem cristais na bile ${ }^{(14,38)}$.

Recentemente, ROSS et al. ${ }^{(37)}$ e LEE et al. ${ }^{(18)}$ demonstraram que microlitíase e "barro biliar" podem ser fatores etiológicos da PAI. O não reconhecimento de tal assertiva induz ao retardo diagnóstico e tratamento apropriado. Não sendo nestes casos, o ultra-som detector sensível ${ }^{(20)} \mathrm{e}$, visto que os microcristais são elementos constituintes daqueles ${ }^{(16)}$, o estudo microscópico da bile assume grande importância por ser técnica confiável ${ }^{(19)}$.

Pelo exposto, percebe-se a importância do estudo de microcristais nas pancreatites, particularmente nas idiopáticas e de etiologia biliar suspeita mas não confirmada. Neste estudo procuramos verificar, em pacientes com pancreatite (idiopática, biliar ou alcoólica), através da análise microscópica da bile do colédoco obtida durante CPRE, a prevalência de microcristais biliares, assim como a sensibilidade e especificidade da sua detecção em indicar etiologia biliar e, principalmente, o significado da detecção de microcristais nos casos de PAI, além da influência da terapêutica específica no índice de recidivas de PAI recorrente, cursando com microcristais.

\section{CASUÍSTICA E METODOLOGIA}

Foram avaliados prospectivamente 60 pacientes com diagnóstico de pancreatite, internados no Hospital São Paulo - Universidade Federal de São Paulo, no período de setembro de 1993 a setembro de 1995. Os pacientes foram divididos em três grupos:

PAI: 20 pacientes não-alcoolistas, sem litíase biliar ao ultrasom, com níveis séricos normais de triglicérides e cálcio, CPRE sem alterações e ausência de outra causa de pancreatite.

PAB: 20 pacientes não-alcoolistas, com achado inequívoco de colelitíase ou coledocolitíase ao ultra-som ou na CPRE, confirmados na cirurgia. 
Pancreatite crônica alcoólica (PCA) agudizada: 20 pacientes com ingestão etílica superior a $80 \mathrm{~g} /$ dia, por 5 anos ou mais, e alterações na CPRE sugestivas de cronicidade (grupo controle).

Como critérios de exclusão, adotou-se qualquer uma das condições predisponentes à precipitação de cristais biliares ${ }^{(20,28,43)}$ : pósoperatório, gravidez, obstrução de vias biliares, hepatopatia, nutrição parenteral prolongada, obesidade com dieta para rápida perda de peso, uso de contraceptivo oral ou octreotide.

O grupo com PAI foi constituído de 7 paciente do sexo masculino e 13 do feminino, com média de idade de 47 anos (variação 18 a 81) e número médio de 2,3 surtos (variação 1-6) de pancreatite por paciente, até inclusão no estudo. Destes pacientes, 11 apresentaram PAI recurrente (dois ou mais episódios de pancreatite). O grupo com $\mathrm{PAB}$ compreendeu 5 pacientes masculinos e 15 femininos, com média de idade de 48,5 anos (variação 18 a 77) e um episódio de pancreatite. O grupo com PCA foi composto de 19 pacientes masculinos e 1 feminino, com média de idade de 39,7 anos (variação 30 a 60) e 2,2 episódios de agudização (variação 1 a 4) de pancreatite por paciente.

O diagnóstico de pancreatite aguda ou crônica agudizada baseouse na associação de dor abdominal aguda, elevação dos níveis sérios de amilase acima três vezes do limite da normalidade e estudo ultrasonográfico ou tomográfico do abdome sugestivo(s) de inflamação pancreática.

Após o tratamento convencional da pancreatite e regressão do processo inflamatório, realizou-se a CPRE com coleta de bile, após jejum de pelo menos 10 horas. Utilizou-se cateter convencional, com diâmetro de $6 \mathrm{Fr}$, para cateterização do ducto biliar comum, sendo seu conteúdo aspirado com seringa estéril de 10 ou $20 \mathrm{~mL}$. Não se utilizou qualquer estímulo para contração da vesícula biliar, tendo-se obtidos $5 \mathrm{~mL}$ de bile para análise em todos os casos. Houve apenas duas complicações pós-CPRE (pancreatite e hemorragia papilar) que se resolveram com medidas clínicas.

As amostras de bile foram imediatamente centrifugadas a $1000 \mathrm{~g}$, durante 10 minutos, e o sedimento aspirado distribuído em duas lâminas. A área inteira de cada lâmina sob a cobertura da lamínula foi examinada em microscopia óptica e de luz polarizada em aumentos de 100X, 200X e 400X, anotando-se a presença ou não de microcristais. Se nesta análise inicial não se detectasse a presença de microcristais, a amostra de bile era incubada em tubo estéril em banho-maria a $37^{\circ} \mathrm{C}$ durante $24 \mathrm{~h}$, recentrifugada e reexaminada da mesma forma inicial.
Os principais tipos de cristais analisados foram MMC e MBC. Os primeiros identificados como estruturas transparentes, de margens paralelas, com forma de paralelogramo e entalhe em uma das bordas, sendo birrefringentes à luz polarizada ${ }^{(14,18)}$; MBC como grânulos amorfos, pequenos, marrom-avermelhados ou amarelo claro a laranja intenso, com tendência à agregação ${ }^{(18,31)}$. As espécimes de bile foram analisadas por dois pesquisadores não conhecedores dos dados clínicos dos pacientes, tendo-se obtido índice de concordância superior a $95 \%$ entre os mesmos, com relação a presença ou não de microcristais. Considerou-se positivo(sedimento anormal) se havia 10 ou mais microcristais por lâmina ou mais que um microcristal por campo em maior aumento (400X), conforme previamente sugerido ${ }^{(14}$, 37). Amostras foram consideradas negativas ou sem significância para cristais, somente se os mesmos não fossem detectados ou fossem escassos (menos que 10 por lâmina) nos dois exames (imediatamente após coleta de bile e após $24 \mathrm{~h}$ de incubação).

Os pacientes com PAB foram colecistectomizados, conforme é rotineiramente realizado. Aqueles com PAI e cristais na bile foram submetidos a colecistectomia eletiva. Naqueles que se recusaram ou eram inaptos à cirurgia, efetuou-se esfincterotomia endoscópica ou tratamento por 6 meses com ácido ursodesoxicólico $(10 \mathrm{mg} / \mathrm{kg} / \mathrm{dia}$, $2 / 3$ da dose após o jantar) via oral, se apenas MMC estivessem presentes $^{(36,37)}$. Nesta última situação, tendo decorridos 6 meses, os pacientes permaneceram em uso de ácido ursodesoxicólico $(300 \mathrm{mg}$ / dia). Nos pacientes com PAI, em que não se detectaram cristais, nenhum tratamento, afora o sintomático, foi realizado. Posteriormente, estes pacientes foram acompanhados em intervalos de pelo menos 3 meses (mínimo de 12 meses), anotando-se a ocorrência ou não de recidivas, qualquer que tenha sido a conduta. Durante o seguimento, foram realizados estudos ultra-sonográficos, com intervalo de 6 meses entre eles, em todos os pacientes nãocolecistectomizados, com a finalidade de observar o aparecimento ou não de colelitíase.

O teste estatístico empregado para comparação da prevalência de microcristais nos três grupos e verificar se a terapêutica específica influenciou na ocorrência de recidivas em pacientes com PAI recurrente e microcristais, foi o teste do qui-quadrado, considerandose como significante $P<0,05$.

O protocolo deste estudo foi aprovado sem restrições pela Comissão de Ética Médica local, tendo-se obtido de todos os pacientes, o termo de consentimento pós-informação. 


\section{RESULTADOS}

prevalência de microcristais na bile do colédoco nas pancreatites

$\mathrm{Na}$ análise da Tabela 1, verifica-se que $75 \%$ dos casos de PAI, $90 \%$ dos pacientes com PAB e apenas $15 \%$ dos casos de PCA, apresentavam um dos tipos de microcristais. Sedimento biliar normal (microcristais ausentes ou escassos - Fig. 1) foi observado na maioria dos casos de PCA, enquanto MMC (Fig. 2, 3) predominaram em todos os grupos em relação aos $\mathrm{MBC}$.

Quando comparados os três grupos, verificou-se que a prevalência de microcristais foi significantemente maior nos grupos com PAI e PAB que naquele com PCA. Por outro lado, não houve diferença estatística significante entre os grupos com PAI e PAB.

TABELA 1 - Prevalência de microcristais na bile do colédoco nas pancreatites

\begin{tabular}{lrrrrrr}
\hline $\begin{array}{l}\text { Tipos de } \\
\text { microcristais }\end{array}$ & PAI $(\mathrm{n}=20)$ & \multicolumn{2}{l}{ PAB $(\mathrm{n}=20)$} & PCA $(\mathrm{n}=20)$ \\
\hline & $\mathrm{n}$ & $\%$ & $\mathrm{n}$ & $\%$ & $\mathrm{n}$ & $\%$ \\
\hline MMC & 13 & 65 & 11 & 55 & 2 & 10 \\
MBC & 0 & 0 & 2 & 10 & 1 & 5 \\
MMC e MBC & 2 & 10 & 5 & 25 & 0 & 0 \\
MMC ou MBC & 15 & 75 & 18 & 90 & 3 & 15 \\
Ausentes & 5 & 25 & 2 & 10 & 17 & 85 \\
\hline PAI - Pancreatite aguda idiopática & & & & \\
PCA - Pancreatite crônica alcoólica & & & & \\
PAB - Pancreatite aguda biliar & \\
MBC - Microcristais de bilirrubinato de cálcio \\
MMC - Microcristais de monohidrato de colesterol
\end{tabular}

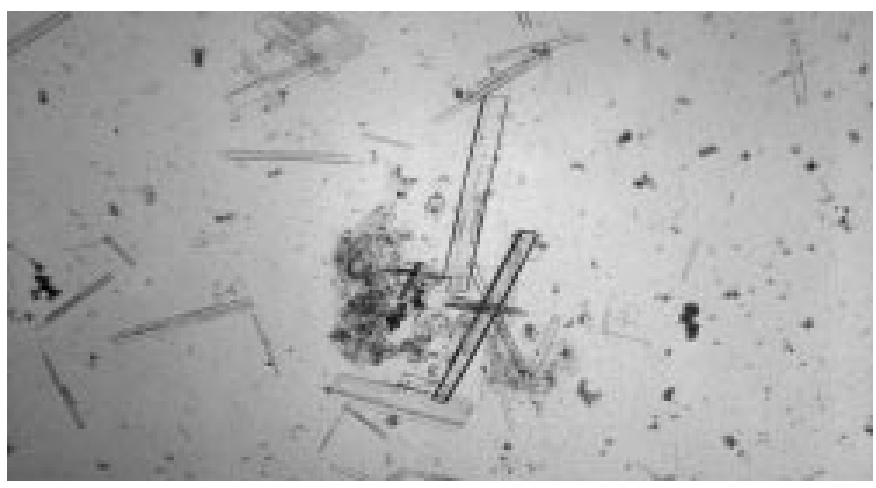

\section{Sensibilidade e especificidade da deteç̧ão de microcristais na bile do colédoco na pancreatite biliar}

Através da análise da Tabela 1, observa-se que a detecção de microcristais para identificação da $\mathrm{PAB}$ apresentou sensibilidade (número de pacientes com PAB que apresentavam MMC ou MBC) de $90 \%$, especificidade (número de pacientes com PCA que não apresentavam MMC ou MBC) de $85 \%$, valor preditivo positivo (probabilidade dos pacientes com microcristais terem etiologia biliar) de $85,7 \%$, valor preditivo negativo (probabilidade dos pacientes sem microcristais não terem etiologia biliar) de $89,4 \%$ e acurácia de $87,5 \%$.

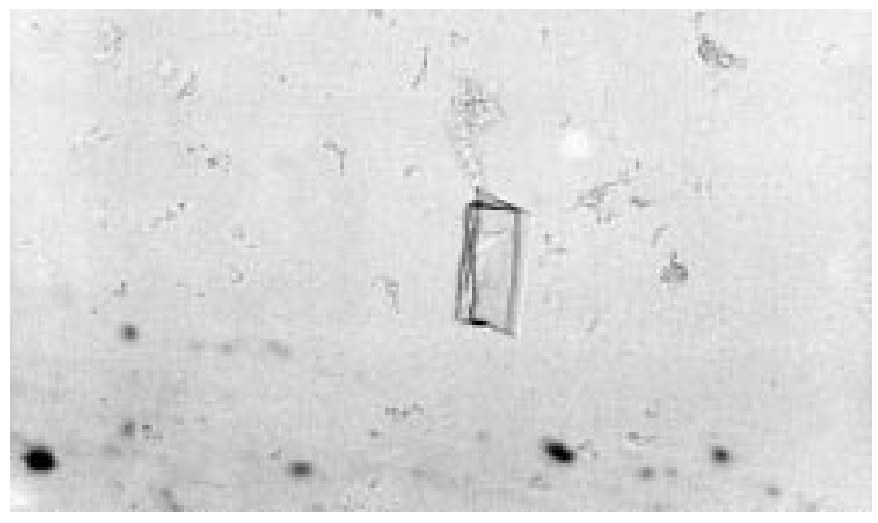

FIGURA 1 - Sedimento biliar normal obtido da bile centrifugada do colédoco em paciente com pancreatite alcoólica. Nota-se, ao centro, um cristal isolado de colesterol com forma característica de paralelogramo (microscopia óptica 200x).

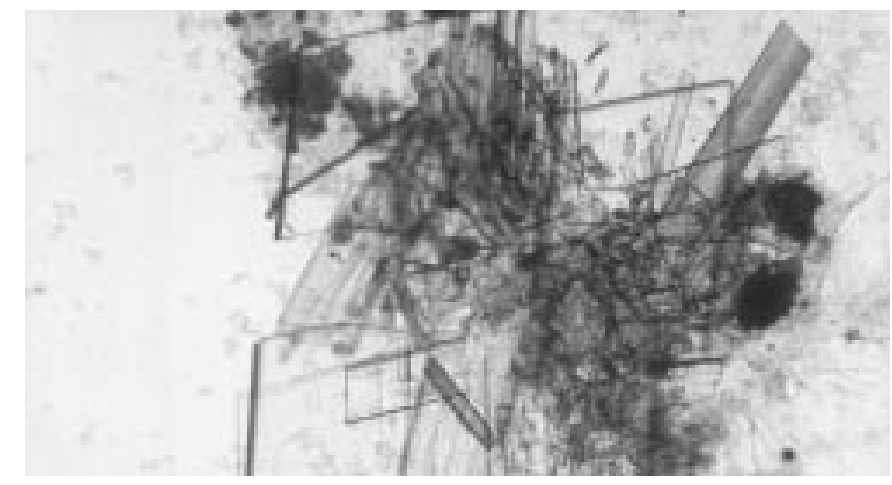

FIGURAS 2 e 3 - Fotomicrografias de diferentes campos do sedimento biliar patológico da bile do colédoco, obtida por colangiopancreatografia endoscópica, em paciente que apresentou seis episódios de pancreatite aguda idiopática. Notam-se agregados de microcristais de monohidrato de colesterol, com margens paralelas e forma típica de paralelogramo (microscopia 400x). 


\section{Evolução clinica, antes e após tratamento específico, dos pa- cientes com PAI recurrente apresentando microcristais na bile}

$\mathrm{Na}$ Tabela 2 encontra-se exposto o número de episódios de pancreatite antes e após tratamento específico dos pacientes com PAI recurrente e microcristais na bile. Através da análise da Tabela 3 , verificou-se que nestes pacientes com PAI recurrente $(n=10)$, apresentando microcristais, houve redução estatisticamente significativa nos episódios de pancreatite após tratamento específico. Desta forma, considerando os próprios pacientes como seus controles, à entrada no estudo, o total de episódios de pancreatite neste subgrupo era de 31 (3,1 por paciente) no intervalo total de tempo de 98 meses (média de 9,8). Após o tratamento específico, observaram-se apenas dois episódios de pancreatite ( 0,2 por paciente) durante o seguimento total de 233 meses (média de 23,3, por paciente) $(P<0,001)$.
Destes 10 pacientes, em $2\left(n^{\circ} 5\right.$ e 6$)$ observou-se novo episódio de pancreatite após tratamento específico (Tabela 2). Num dos casos, recidiva ocorreu 2 meses após colecistectomia. Nesta ocasião, CPRE sugeriu a presença de papilite e identificou um cálculo do colédoco de $4 \mathrm{~mm}$. Após esfincterotomia endoscópica e extração do cálculo, nenhum episódio adicional de pancreatite ocorreu nos 22 meses subseqüentes. No outro paciente, recurrência foi observada 3 meses após a colecistectomia. Análise da bile obtida por CPRE, nesta ocasião, revelou persistência de MMC. Efetuou-se esfincterotomia endoscópica complementar e, durante 20 meses seguintes, nenhum episódio de pancreatite foi registrado.

No estudo anatomopatológico da peça cirúrgica, todos os quatro pacientes submetidos a colecistectomia apresentavam colecistite crônica e em uma das peças notaram-se três microcálculos de $2 \mathrm{~mm}$.

TABELA 2 - Evolução clínica, antes e após tratamento, dos pacientes com pancreatite aguda recurrente idiopática apresentando microcristais biliares

\begin{tabular}{|c|c|c|c|c|c|c|}
\hline & Pac & $\begin{array}{l}\text { Episódios* de } \\
\text { pancreatite }\end{array}$ & $\begin{array}{c}\text { Intervalo** } \\
(\operatorname{meses})\end{array}$ & Tratamento & $\begin{array}{c}\text { Tempo de } \\
\text { seguimento (meses) }\end{array}$ & Recidivas*** \\
\hline & 1 & 3 & 6 & Ac. ursodesoxicólico & 24 & Nenhuma \\
\hline & 2 & 3 & 3 & Ac. ursodesoxicólico & 34 & Nenhuma \\
\hline & 3 & 2 & 3 & Papilotomia & 26 & Nenhuma \\
\hline & 4 & 2 & 4 & Ac. ursodesoxicólico & 25 & Nenhuma \\
\hline & 5 & 6 & 24 & Colecistectomia/poste- & 24 & 1 \\
\hline & & & & riormente papilotomia & & \\
\hline & 6 & 3 & 12 & Colecistectomia/poste- & 23 & 1 \\
\hline & & & & riormente papilotomia & & \\
\hline & 7 & 3 & 24 & Ac. ursodesoxicólico & 23 & Nenhuma \\
\hline & 8 & 3 & 4 & Colecistectomia & 19 & Nenhuma \\
\hline & 9 & 3 & 6 & Colecistectomia & 18 & Nenhuma \\
\hline & 10 & 3 & 12 & Papilotomia & 17 & Nenhuma \\
\hline Total & 10 & 31 & 98 & & 233 & 2 \\
\hline Média & - & $3,1 \pm 1,1$ & $9,8 \pm 8,2$ & & $23,3 \pm 4,8$ & 0,2 \\
\hline
\end{tabular}

TABELA 3 - Comparação dos totais do tempo de seguimento e número de episódios de pancreatite antes e depois do tratamento, em pacientes com pancreatite aguda recurrente idiopática $(n=10)$

\begin{tabular}{lc}
\hline & Tempo (meses) \\
\hline Antes do tratamento & 98 \\
Depois do tratamento & 233 \\
\hline $\begin{array}{l}\text { Teste do qui-quadrado } \\
\chi^{2} \text { calculado }=51,95^{*}\end{array}$ & $\chi^{2}$ crítico $=3,84$
\end{tabular}


TABELA 4 - Evolução clínica, antes e após tratamento, dos pacientes com pancreatite aguda idiopática (não-recurrente) apresentando microcristais biliares $(n=5)$

\begin{tabular}{|c|c|c|c|c|}
\hline Pac & $\begin{array}{l}\text { Episódios* de } \\
\text { pancreatite }\end{array}$ & Tratamento & $\begin{array}{c}\text { Tempo de } \\
\text { seguimento (meses) }\end{array}$ & Recidivas** \\
\hline 11 & 1 & Ac. ursodesoxicólico & 30 & Nenhuma \\
\hline 12 & 1 & $\begin{array}{l}\text { Ac.ursodesoxicólico/poste- } \\
\text { riormente papilotomia }\end{array}$ & 23 & 1 \\
\hline 13 & 1 & Papilotomia & 20 & Nenhuma \\
\hline 14 & 1 & Ac. ursodesoxicólico & 20 & Nenhuma \\
\hline 15 & 1 & Colecistectomia & 12 & Nenhuma \\
\hline
\end{tabular}

* Número de episódios de pancreatite até a entrada no presente estudo

** Número de recidivas durante o seguimento após tratamento específico

\section{Evolução clínica, antes e após tratamento específico, dos pacientes com PAI (não-recurrente) apresentando micro- cristais na bile $(n=5)$}

Pela análise da Tabela 4, verifica-se que durante o seguimento médio de 21 meses após tratamento, novo episódio de pancreatite ocorreu em um dos casos, após 6 meses de uso oral de ácido ursodesoxicólico. Nova avaliação por CPRE e análise da bile demonstrou persistência de MMC. Optou-se por efetuar esfincterotomia endoscópica e, durante 17 meses seguintes, não se notou recidiva de pancreatite. Estudos ultra-sonográficos subseqüentes revelaram o aparecimento de colelitíase em um dos pacientes, 12 meses após o episódio inicial de pancreatite. Além disso, colecistite crônica e colesterolose foram observados no estudo anatomopatológico do paciente submetido a colecistectomia.

\section{Evolução clínica, antes e após a entrada no estudo, dos pacientes com PAI sem microcristais na bile $(n=5)$}

Durante o seguimento médio de 18 meses no grupo com PAI sem microcristais, um paciente apresentou dois novos episódios de pancreatite. CPRE e análise da bile nestas ocasiões, não revelaram qualquer anormalidade. Estudo ultra-sonográfico durante este período não detectou colelitíase em nenhum destes cinco casos.

\section{DISCUSSÃO}

No presente estudo, bile para análise de microcristais em pacientes com pancreatite foi obtida durante CPRE, sem a utilização de qualquer estímulo químico para contração da vesícula biliar. Admitindo-se que a colecistocinina, por estimular a secreção pancreática, seja contraindicada nas primeiras semanas de evolução da pancreatite ${ }^{(19)}$ e tendo em vista o fato da CPRE ser investigação praticamente obrigatória na suspeita de PAB ou PAI, torna-se evidente que a coleta da bile, para análise de microcristais, pode ser feita mais precocemente e durante a própria CPRE. Isto seria mérito do procedimento, visto que pacientes com patologia biliar oculta, que apresentam grande risco de recurrências de pancreatite ${ }^{(18,37)}$, seriam mais prontamente identificados e tratados. Deste modo, reduzir-se-ia o risco de novo episódio de pancreatite, se comparado ao paciente que aguardasse a realização de tubagem duodenal, para os mesmos propósitos que, por exigir estímulo químico para contração da vesícula biliar, é realizada mais tardiamente, entre 4 e 8 semanas após o episódio de pancreatite ${ }^{(18,37)}$.

$\mathrm{Na}$ presente casuística, a detecção de microcristais teve alta sensibilidade em identificar pacientes com PAB. Os prováveis fatores responsáveis por este fato incluem:

- a bile do colédoco não é diluída por secreção gástrica, intestinal ou pancreática;

- melhor otimização no processamento e análise da bile, utilizando a centrifugação (permitindo a concentração de cristais), a incubação das amostras por $24 \mathrm{~h}$ (detectando a eventual ocorrência de cristalização retardada da bile) e a microscopia de luz polarizada que aumenta a detecção de $\operatorname{MMC}^{(6,17,19)}$.

Esta alta sensibilidade (90\%) contrasta com estudos anteriores que relataram taxas de $57 \%$ a $70 \%$ de identificação de microcristais na bile duodenal ${ }^{(14,23,44)}$, em pacientes com cálculos biliares. Isto sugere que para o estudo microscópico da bile, visando a identificação de microcristais, os métodos de coleta, de análise e processamento de bile utilizados neste estudo, são bastante satisfatórios e superiores àqueles empregados na tubagem duodenal.

Através da análise da Tabela 1, pode-se determinar indiretamente em que extensão pacientes com PAB e PCA são distinguidos pela análise de microcristais. Assim, MMC e/ou MBC estavam presentes em 18 de 20 pacientes com PAB (sensibilidade 90\%) e em apenas 3 dos 20 casos de PCA (especificidade 85\%). Destarte, poder-se-ia afirmar que a presença de microcristais na bile do colédoco em paciente com PA, assinala provável etiologia biliar subjacente. De interesse, refere-se ao achado de MMC em um paciente com $\mathrm{PAB}$, apenas após incubação da bile por $24 \mathrm{~h}$. Este mesmo fato foi observado em um dos pacientes com PAI, que provou ter colecistite crônica e colesterolose 
no estudo anatomopatológico. Por outro lado, nenhum dos pacientes com PCA, sem microcristais na análise imediata, desenvolveu cristais após incubação da bile por $24 \mathrm{~h}$, confirmando que formação retardada de cristais é característica da bile litogênica ${ }^{(38)}$. Este fenômeno referenda a importância de se proceder à incubação da bile em todos os casos onde a análise imediata para microcristais resultar negativa.

A alta prevalência de microcristais na bile do colédoco observada nos pacientes com PAB e PAI deve ser confiável, visto que se procurou excluir, através da história, dos métodos de imagem e da CPRE, condições predisponentes à formação destes, ou seja, que ocasionam estase na vesícula biliar ${ }^{(20,28,43)}$. Por outro lado, cristais foram incomuns na PCA, sugerindo que os mesmos não são secundários à inflamação pancreática. Nos três casos de PCA em que microcristais estavam presentes, o achado de MBC poderia ser atribuído ao efeito do álcool que, aumentando as concentrações biliares de bilirrubina não-conjugada, facilitaria a precipitação de bilirrubinato de cálcio ${ }^{(5)}$. Por sua vez, é plausível que os dois pacientes com MMC tenham além da PCA, bile litogênica.

Os seguintes achados consubstanciam a hipótese de que a presença de cristais na PAI represente indício de que estas sejam formas frustas de PAB e não verdadeiramente idiopáticas:

- a prevalência de microcristais foi semelhante em pacientes com PAI e PAB;

- todos os pacientes colecistectomizados apresentavam alterações patológicas na peça cirúrgica (colecistite crônica nos cinco, além de microlitíase em um dos casos);

- durante o seguimento, o aparecimento de colelitíase foi notado em um paciente e cálculo de colédoco foi detectado em outro, durante recidiva de pancreatite;

- a substancial redução das recidivas de pancreatite nos pacientes com PAI recurrente e microcristais notada após terapêutica para patologia biliar;

- recurrência de pancreatite após tratamento específico nos pacientes com cristais na bile só ocorreu nos casos que apresentavam "fator biliar persistente" (papilite, microcristais ou litíase no colédoco).

A redução dos episódios de PA durante o seguimento, notada nos pacientes com PAI recurrente após tratamento específico (Tabela $3)$, reitera resultados de estudos anteriores ${ }^{(18,37)}$ e merece comentários. O efeito benéfico do ácido ursodesoxicólico deve-se, provavelmente, ao seu emprego apenas em pacientes que apresentavam MMC, sabidamente passíveis à dissolução por sais biliares $^{(36,37)} \mathrm{e}$ ao fato da maior parte dos pacientes estarem ainda em estágio precoce do desenvolvimento de cálculos de colesterol e ainda não apresentarem litíase biliar macroscópica (que seria mais resistente à dissolução ou que exigiria tempo mais prolongado de tratamento se comparado aos agregados de microcristais). Ressalte-se que, provavelmente, o uso do ácido ursodesoxicólico, nestas circuns- tâncias, deverá ser feito por tempo indeterminado, visto que as condições de litogênese, sem a administração do sal biliar, permanecerão inalteradas a longo prazo. Por sua vez, a papilotomia elimina o canal comum ${ }^{(39)}$, além de impedir a impactação na região ampular, de aglomerados de cristais ou microcálculos porventura existentes. Secundando tal especulação, cita-se que nenhum dos cinco pacientes submetidos a papilotomia desenvolveu novo episódio de pancreatite durante o seguimento. Além disso, em dois estudos recentes ${ }^{(39,45)}$ nos quais esfincterotomia endoscópica foi realizada com o intuito de evitar recidivas de $\mathrm{PAB}$, em pacientes de alto risco cirúrgico, observou-se que a recurrência de pancreatite foi um raro evento durante o seguimento.

Outra modalidade terapêutica utilizada foi a colecistectomia, cujo benefício deve-se à eliminação do principal local de formação e crescimento de microcristais e cálculos biliares ${ }^{(41)}$. Salienta-se que mesmo sua retirada pode não impedir a eventual recidiva da pancreatite, conseqüente à migração de cálculo no colédoco não detectado durante a cirurgia, ou da circunstância incomum de formação e persistência de cristais na bile hepática ${ }^{(12)}$, como documentado em um dos casos. Em ambas as situações, esfincterotomia complementar impediu a ocorrência de novos episódios de PA.

Outro aspecto conspícuo observado, refere-se ao achado histológico de colecistite crônica nos cinco pacientes com PAI e microcristais submetidos a colecistectomia, apesar da ausência de sintomas atribuídos ao trato biliar em todos os casos. Este achado corrobora a hipótese que patologia oculta do trato biliar pode estar presente em casos de PAI recurrente, sendo o único indício de sua presença nesta circunstância, um sedimento biliar contendo microcristais $^{(10)}$.

Em relação ao(s) mecanismos(s) putativo(s) de pancreatite nos pacientes com PAI e microcristais, algumas conjecturas podem ser feitas. É admissível que pelo menos alguns casos fossem reminiscência de PAB típica, pela possibilidade de migração precoce transampular do cálculo e sua posterior eliminação, não havendo litíase residual na época da avaliação ${ }^{(27)}$. Por outra ótica, microagregados de cristais ou microcálculos, identificados apenas pela análise microscópica da bile, poderiam impactar transitória e repetidamente, ocasionando espasmo (obstrução) e/ou inflamação da papila e, conseqüentemente, pancreatite aguda.

Não obstante, o fato de cristais estarem ausentes ou raramente serem encontrados na bile de indivíduos normais ${ }^{(14,23)}$, obviamente que a simples presença de microcristais não implica em risco de PA. Destarte, muitos portadores de microcristais eliminá-los-ão para o duodeno sem conseqüências. Diferenças na físiologia e/ou anatomia da região ampular poderiam predispor alguns pacientes com microcristais ao desenvolvimento de pancreatite. Postula-se que pancreatite ocorreria nos que apresentassem canal biliopancreático comum estreito $^{(1,8,39)}$. 
Sabendo-se que nucleação e precipitação de cristais podem ser estágios efêmeros ${ }^{(40)}$, com rápida evolução para cálculo macroscópico, outra inferência é que a presença de microcristais em pacientes com PAI tenha o mesmo significado fisiopatológico do cálculo biliar. De forma sintética, poder-se-ia dizer que microcristais em pacientes com PAI denotaria não apenas um estágio precoce na formação do cálculo biliar, mas também permitiria identificar os pacientes com cálculos não-detectáveis por outros métodos convencionais, ambas situações nas quais o risco para novos ataques de PA é considerável ${ }^{(27)}$.

É notório que a proporção das pancreatites idiopáticas encontrase em razão inversa da profundidade da metodologia diagnóstica empregada. Neste plano, demonstramos que a análise de microcristais na bile obtida durante CPRE, representa importante contribuição, revelando formas "ocultas" ou "frustas" de PAB, em proporção significante dos casos de pancreatites rotuladas de idiopáticas $(2 / 3$ dos pacientes).

Finalmente, poder-se-ia afirmar que na PAI, a coleta de bile durante CPRE, visando a análise e detecção de microcristais, é método prático, seguro, objetivo, sensível e específico para identificação de etiologia biliar subjacente oculta. Isto permite intervenções terapêuticas resolutivas precoces (colecistectomia, esfincterotomia endoscópica ou uso de ácido ursodesoxicólico), que reduzem o risco de recidivas de pancreatite. Parece também evidente que PA (particularmente, recurrente) não deveria ser rotulada como idiopática antes da análise microscópica da bile visando detecção ou exclusão de microcristais.

Chebli JMF, Ferrari Jr AP, Silva MRR, Borges DR, Atallah AN, Neves MM das. Biliary microcrystals in idiopathic acute pancreatitis: clue for occult underlying biliary etiology. Arq Gastroenterol 2000;37(2):93-101.

ABSTRACT - The main causes of pancreatic inflammation worldwide are biliary lithiasis and alcoholism. However, 10 to $30 \%$ of patients have been considered to have "idiopathic" acute pancreatitis. Recently, some studies showed that a significant rate of the so called idiopathic pancreatitis are caused by microlithiasis and/or biliary sludge, identified by the presence of cholesterol monohidrate and/or calcium bilirubinate microcrystals in the biliary sediment. In the present study, the analysis of microcrystals from bile obtained during endoscopic retrograde cholangiopancreatography was done in patients with pancreatitis (idiopathic, biliary or alcoholic-20 in each group). Patients with idiopathic pancreatitis and microcrystals in the bile underwent cholecystectomy whenever possible. Those who refused or were inapt to surgery underwent endoscopic sphincterotomy or received continuous therapy with ursodeoxycholic acid. Patients with idiopathic pancreatitis without biliary crystals did not receive any specific treatment. The prevalence of biliary microcrystals in patients with idiopathic pancreatitis (75\%) and biliary pancreatitis (90\%) was significantly higher than in those with alcoholic pancreatitis (15\%). In the identification of the etiology of biliary pancreatitis, the presence of microcrystals had a sensitivity of $90 \%$, specificity of $85 \%$, positive predictive value of $85,7 \%$, negative predictive value of $89,4 \%$ and accuracy of $87,5 \%$. In the patients with recurrent idiopathic pancreatitis, with biliary crystals, there was an statistically significant reduction in the number of pancreatitis episodes after specific treatment. In the follow-up of this group during 23,3 \pm 4,8 months, recurrence of pancreatitis occurred only in patients with "persistent biliary factor" (choledocholithiasis and/or persistence of cholesterol monohidrate). All patients with idiopathic pancreatitis who underwent cholecystectomy had chronic cholecystitis. Moreover, cholelithiasis was present in one case. In the ultrassonographic follow-up of the patients with idiopathic acute pancreatitis with microcrystals in the bile, cholelithiasis was detected in one case. In the subgroup of five patients with idiopathic pancreatitis without biliary microcrystals recurrence occurred in one case. Ultrassonographic study during follow-up did not reveal biliary stones in any of these patients. We concluded that the detection of biliary microcrystals in "idiopathic" pancreatitis suggested an underlying biliary etiology, even if occult. What's more, early specific therapeutic procedure (cholecystectomy, endoscopic sphincterotomy or ursodeoxycholic acid) in patients with recurrent idiopathic pancreatitis with microcrystals in the bile reduced significantly the recurrence during the followup. Finally, acute pancreatitis (specially recurrent) should not be called idiopathic before the microscopic analysis of the bile, aiming to detect or exclude the presence of microcrystals.

HEADINGS - Pancreatitis. Cholelithiasis. Cholecistitis. Bile. Biliary sludge. 


\section{REFERÊNCIAS BIBLIOGRÁFICAS}

1. Acosta JM, Pellegrini CA, Skinner DB. Etiology and pathogenesis of acute biliary pancreatitis. Surgery 1980;88:118-25

2. Burrel MI, Avella J, Spiro HM, Taylor KJW. Diagnostic imaging procedures in acute pancreatitis. JAMA 1979;242:342-6.

3. Carter DC. Gallstone pancreatitis. Br Med J 1983;286:103-4.

4. Delchier JC, Benfredj P, Preaux AM, Metreau JM, Dhumeaux D. The usefulness of microscopic bile examination in patients with suspected microlithiasis: a prospective evaluation. Hepatology 1986;6:118-22.

5. Di Padova C, Tritapepe R, Rovagnati P, Bessone E, Di Padova F. Effect of ethanol on biliary unconjugated bilirrubin and it's implication in pigment gallstone pathogenesis in humans. Digestion 1982;24:112-7.

6. Donovan JM, Carey MC. Physical-chemical basis of gallstone formation. Gastroenterol Clin North Am 1991;20:47-66.

7. Farinon AM, Ricci GL, Sianesi M, Percudani M, Zanella E. Physiopathologic role of microlithiasis in gallstone pancreatitis. Surg Gynecol Obstet 1987; $164: 252-6$

8. Flati G, Flati D, Porowska B, Ventura T, Catarci M, Carboni M. Surgical anatomy of the papilla of valer and biliopancreatic ducts. Am Surg 1994;60:712-8

9. Freund H, Pfeffermann R, Durst AL, Rabinovici N. Gallstone pancreatitis: explorations of the biliary system in acute and recurrent pancreatitis. Arch Surg 1976;111:1106-7.

10. Goldstein F, Kucer FT, Thornton JJ, Abramson J. Acute and relapsing pancreatitis caused by bile pigment aggregates and diagnosed by biliary drainage. Am J Gastroenterol 1980;74:225-8.

11. Goodman AJ, Neoptolemos JP, Carrlocke DL, Finlay DBL, Fossard DP. Detection of gallstones after acute pancreatitis. Gut 1985;26:125-32

12. Grier JF, Cohen SW, Grafton WD, Gholson CF. Acute suppurative cholangitis associated with choledochal sludge. Am J Gastroenterol 1994;89:617-9.

13. Houssin D, Castaing D, Lemoine J, Bismuth H. Microlithiasis of the gallbladder Surg Gynecol Obstet 1983;157:20-4.

14. Juniper K, Burson EN. Biliary tract studies. II. The significance of biliary crystals. Gastroenterology 1957;32:175-211.

15. Kelly TR, Swaney PE. Gallstone pancreatitis: the second time around. Surgery 1982;92:571-5

16. Lee SP, Maher K, Nicholls JF. Origin and fate of biliary sludge. Gastroenterology 1988;94:170-6.

17. Lee SP. Pathogenesis of biliary sludge. Hepatology 1990;12:200S-5S

18. Lee SP, Nicholls JF, Park HZ. Biliary sludge as a cause of acute pancreatitis. N Engl J Med 1992;326:589-93.

19. Lee SP. Biliary sludge and acute pancreatitis. Hosp Pract 1994;29:45-52

20. Lee SP, Hayashi A, Kim YS. Biliary sludge: curiosity or culprit? Hepatology 1994;20:523-5.

21. López RA, Fugarolas GM, Rodero GC, Rodriguez EP, Alvarez JLM, Cabrera D. Valor del sondaje duodenal en el diagnóstico etiológico de la pancreatitis aguda. Rev Esp Enf Dig 1993;83:363-6.

22. Lyon BBV. Diagnosis and treatment of diseases of the gallbladder and biliary ducts; preliminary report on a new method. JAMA 1919;73:980-2.

23. Marks JW, Bonorris G. Intermittency of cholesterol crystals in duodenal bile from gallstone patients. Gastroenterology 1984;87:622-7.

24. Marshall JB. Acute pancreatitis. A review with an emphasis on new developments Arch Intern Med 1993;153:1185-98.

25. Mayer AD, McMahon MJ. Gallstones and acute pancreatitis - is the association underestimated? (Abstract). Br J Surg 1984;71:905.
26. Neoptolemos JP, Hall AW, Finlay DF, Berry JM, Carr-Locke DL, Fossard DP. The urgent diagnosis of gallstones in acute pancreatitis: a prospective study of three methods. Br J Surg 1984;71:230-3.

27. Neoptolemos JP, Davidson BR, Winder AF, Vallance D. Role of duodenal bile crystal analysis in the investigation of idiopathic pancreatitis. $\mathrm{Br} \mathrm{J}$ Surg $1988 ; 75: 450-3$

28. Ohara N, Schaefer J. Clinical significance of biliary sludge. J Clin Gastroentero 1990;12:291-4.

29. Paloyan D, Simonowitz D, Skinner D. The timing of biliary tract operations in patients with pancreatitis associated with gallstones. Surg Gynecol Obstet 1975;141:737-9.

30. Parikh NJ, Geenen JE. Current role of ERCP in the management of benign pancreatic disease. Endoscopy 1992;24:120-4.

31. Rafsky HA. Diagnostic importance of biliary crystals. Am J Med Sci $1933 ; 185: 851$

32. Ramond MJ, Dumont M, Belghiti J, Erlinger S. Sensitivity and specificity of microscopic examination of gallbladder bile for gallstone recognition and identification. Gastroenterology 1988;95:1339-43.

33. Ranson JHC. The timing of biliary surgery in acute pancreatitis. Ann Surg 1979;189:654-63

34. Reber HA. Idiopathic pancreatitis: management of the patient with recurrent pancreatitis. In: Howard JM, Jordan GL, Reber HA, editors. Surgical diseases of the pancreas. 2.ed. Philadelphya: Lea \& Febiger; 1987. p.348-52.

35. Reisberg IR, Mabee GW. Endoscopic biliary drainage for detection of gallbladder disease. Gastrointest Endosc 1979;25:6-9.

36. Ross E, Navarro S, Fernandez I, Reixach M, Ribó JM, Rodés J. Utility of biliary microscopy for the prediction of the chemical composition of gallstones and the outcome of dissolution therapy with ursodeoxycholic acid. Gastroenterology 1986;91:703-12.

37. Ross E, Navarro S, Bru C, Garcia-Pugés A, Valderrama R. Occult microlithiasis in "idiopathic" acute pancreatitis: prevention of relapses by cholecystectomy or ursodeoxycholic acid therapy. Gastroenterology 1991;101:1701-9.

38. Sedaghat A, Grundy SM. Cholesterol crystals and the formation of cholestero gallstones. N Engl J Med 1980;302:1274-7.

39. Siegel JH, Veerappan A, Cohen SA, Kasmin FE - Endoscopic sphincterotomy for biliary pancreatitis: an alternative to cholecystectomy in high - risk patients Gastrointest Endosc 1994;40:573-5.

40. Small DM. Nucleation and growth of cholesterol gallstones. Méd Chir Dig 1980;9:619-23.

41. Smith BF, Lamont JT. Bovine gallbladder mucin binds bilirubin in vitro. Gastroenterology 1983;85:707-12.

42. Steinberg W, Tenner S. Acute pancreatitis. N Engl J Med 1994;330:1198-210.

43. Strasberg SM, Toth JL; Gallinger S, Harvey PRC. High protein and total lipid concentration are associated with reduced metastability of bile in an early stage of cholesterol gallstone formation. Gastroenterology 1990;98:739-46.

44. Susann PW, Sheppard F, Baloga AJ. Detection of occult gallbladder disease by duodenal drainage collected endoscopically. A clinical and pathologic correlation. Am Surg 1985;51:162-5.

45. Welbourn CRB, Beckly DE, Eyrebrook IA. Endoscopic sphincterotomy without cholecystectomy for gallstone pancreatitis. Gut 1995;37:119-20.

Recebido para publicação em 27/7/1999. Aprovado para publicação em 20/12/1999. 